\title{
Kompetenzmodellierungen für den Mathematikunterricht - Eine Zwischenbilanz aus Sicht der Mathematikdidaktik
}

\author{
Rolf Biehler • Timo Leuders
}

Online publiziert: 23. Januar 2014

(C) GDM 2014

Seit inzwischen über einem Jahrzehnt ist dem Begriff „Kompetenz“ aus den Vergleichsstudien der empirischen Bildungsforschung (Baumert et al. 2001) und den Standardsetzungen der Bildungspolitik (vgl. Klieme et al. 2003) eine hohe Präsenz beschieden. Theoretischer Bezugspunkt ist dabei meist die pädagogische Psychologie (Weinert 2001), welche das Kompetenzkonzept für das Lernen und Leisten an allgemeinbildenden Schulen aufbereitet hat. In der Mathematikdidaktik spielt der Kompetenzbegriff im Zusammenhang mit Fragen mathematischer Bildung eine zentrale Rolle und wird zunehmend im Rahmen von fachspezifischen empirischen Forschungen weiter ausdifferenziert (vgl. Leuders 2011). Es ist charakteristisch für die Breite des Aufgabenbereichs der Mathematikdidaktik, dass Kompetenzkonzepte auf einer theoretischen, einer forschungsmethodologischen, sowie auf einer bildungs- und forschungspolitischen Ebene bearbeitet werden. Im vorliegenden Themenschwerpunkt des Journal für Mathematikdidaktik finden sich hierzu Beiträge, die die genannten Ebenen auf verschiedene Weise verbinden. Dabei haben wir insbesondere Wissenschaftlerinnen und Wissenschaftler zu einem Beitrag eingeladen, die im Rahmen des Schwerpunktprogrammes der Deutschen Forschungsgemeinschaft „Kompetenzmodelle zur Erfassung individueller Lernergebnisse und zur Bilanzierung von Bildungsprozessen“ (2007-2012, Klieme und Leutner 2006) eigene Projekte durchgeführt haben.

R. Biehler ( $\varangle)$

Institut für Mathematik, Universität Paderborn, Warburger Str. 100, 33098 Paderborn, Deutschland e-mail: biehler@math.upb.de

T. Leuders

Institut für Mathematische Bildung Freiburg, IMBF - Pädagogische Hochschule Freiburg,

Kunzenweg 21, 79117 Freiburg, Deutschland

e-mail: leuders@ph-freiburg.de 
Aus theoretischer Perspektive ist der Kompetenzbegriff für die Fachdidaktiken von hoher Attraktivität, da er im Gegensatz zu allgemeinpsychologischen Konzepten, wie z.B. der Intelligenz oder der Motivation, Aspekte hervorhebt, die eine hohe Affinität mit einer fachspezifischen Sicht auf menschliches Können und Lernen besitzen: Kompetenzen sind immer situations- bzw. kontextbezogen zu verstehen, und damit insbesondere für ein Schulfach oder sogar bestimmte Teilgebiete spezifisch zu betrachten (Niss 2003; Hartig et al. 2008). Das Kompetenzkonstrukt scheint also besonders geeignet, die „lokalen Theorien“, welche die Fachdidaktik zu vielen relevanten Inhaltsbereichen mathematischen Lernens und Leistens entwickelt und empirisch fundiert hat, zu beschreiben. Eine fachdidaktische Auseinandersetzung mit dem Kompetenzkonstrukt bedeutet folglich auch, Bezüge zu bisherigen fachdidaktischen Forschungen herzustellen und ihren Mehrwert zu untersuchen. Eine Reihe von fachdidaktischen Projekten der letzte Dekade werden im Beitrag von Leuders in diesem Heft diskutiert. Ebenfalls im vorliegenden Heft befassen sich Buchholtz, Kaiser und Blömeke im Anschluss an die aktuelle Forschung zur Struktur professioneller Kompetenzen mit der theoretischen Konzeptualisierung von Professionswissen von Mathematiklehrenden und finden mittels einer Kompetenzmessung empirische Hinweise auf Bezüge zwischen mathematikdidaktischem und erziehungswissenschaftlichem Wissen.

Eine Beschäftigung mit Kompetenzen ist oft auch mit einer methodologischen Perspektive verbunden. Die im Zusammenhang mit der am Kompetenzkonstrukt orientierten Forschung der letzten Jahre entwickelten statistischen Ansätze, allen voran die probabilistischen Testmodelle, bieten vielfältige methodische Möglichkeiten, postulierte Fähigkeitsstrukturen empirisch abzubilden und überprüfbar zu machen, welche mit klassischer Testtheorie nicht möglich waren (Rost 2004). Dabei gehen die Möglichkeiten über die im Zusammenhang mit internationalen Leistungsvergleichsstudien bekannten und oft wegen ihrer Eindimensionalität kritisierten Ansätze vergleichender Kompetenzmessung (z.B. OECD 1999) hinaus. Die verfügbare Vielfalt an psychometrischen Modellen macht es möglich, eine große Breite an Theorien über Charakteristika und Zusammenhänge von Teilfähigkeiten modelltheoretisch abzubilden und empirisch zugänglich zu machen (z.B. Hartig 2008; Rupp et al. 2010). Mit Blick auf die Fortschritte in Fachdidaktik und Psychometrie formulieren schon Pellegrino et al. (2001), dass bei diesen Forschungsunternehmungen Verfahren der Leistungsmessung besser in Einklang mit Theorien über kognitive Strukturen zu bringen seien. Das oben bereits erwähnte DFG-Schwerpunktprogramm sollte hier den Rahmen bieten, die Möglichkeiten und Grenzen solcher Kompetenzmodellierungen im Bereich des allgemeinbildenden Schulwesens auszuloten. Dabei bot sich eine besondere Chance für die enge Kooperation von Expertinnen und Experten aus dem Bereich der Pädagogischen Psychologie, der Psychometrie und der Fachdidaktik, welche in einigen Teilprojekten angegangen wurde (Biologie-, Chemie-, Physik-, Geographie-, Deutsch- und Mathematikdidaktik). Der Beitrag von Leuders in diesem Heft zeigt auf, in welcher Weise im Bereich des Faches Mathematik innerhalb und außerhalb des Schwerpunktprogrammes eine solche Zusammenarbeit zur Entwicklung valider Kompetenzmodelle unterschiedlichster Struktur beigetragen hat. Der Beitrag von Lingel, Neuenhaus, Artel und Schneider in diesem Heft wiederum entwickelt und nutzt ein neues Instrument zur Erfassung metakognitiven Wissens, 
welches durch die Einbeziehung fachdidaktischer Expertise auf Lernende der fünften Jahrgangsstufe und speziell auf das Fach Mathematik zugeschnitten wurde.

Das Konzept der Kompetenz hat nicht nur die Forschung beschäftigt, sondern vielleicht weit mehr noch die Bildungspolitik und alle Ebenen des allgemeinbildenden Schulsystems (Klieme et al. 2003). Der Fokus lag dabei aber weniger auf der grundlegenden theoretischen Beschreibung und empirischen Erfassung von Schülerfähigkeiten in bestimmten Inhaltsbereichen als auf dem Ausloten der Möglichkeiten eines für Deutschland neuen Steuerungsparadigmas (Altrichter und Heinrich 2006). Mit der Bezeichnung „Kompetenz“ war dabei - jenseits aller wissenschaftlich differenzierten Definitionsversuche - bei den bildungspolitischen Entscheidungsträgern die Hoffnung verbunden, dass man sich von den als kaum erreichbar wahrgenommenen Inhaltskatalogen lösen und sich darauf konzentrieren konnte, was Lernende wirklich können sollten (Stichwort: „Kerncurriculum“, vgl. Böttcher 2002). Der in der Nachfolge der PISA-Rezeption intensiver werdende curriculare Diskurs (der in der Mathematikdidaktik schon länger geführt wurde, vgl. z.B. Heymann 1996), hat dazu geführt, dass allgemeine Kompetenzen wie Problemlösen und Modellieren im Vergleich zu inhaltlichem mathematischen Wissen wieder stärker in das Bewusstsein gerückt wurden (Leuders et al. 2005). Dieser Aspekt von Kompetenzorientierung wurde auch zu einem kritisches Instrument, mit dem ein vorwiegend an technischen Fertigkeiten orientierter Unterricht in seinen Begrenzungen auf neue Weise diskutierbar gemacht wurde. Die Wirkungen auf die Praxis sind allerdings nicht in wenigen Jahren zu bemessen, möglicherweise wird der Mathematikunterricht auf längere Sicht sich nicht fundamental ändern. Einen entscheidenden Einfluss können dabei die gleichzeitig mit neuen Standardsetzungen eingeführten Instrumente der Standardüberprüfung haben, vor allem in Form zentraler Lernstandserhebungen und Vergleichsarbeiten (Helmke et al. 2004). Dies ist der Bereich, in denen die Praxis bislang am stärksten mit Kompetenzmodellierungen konfrontiert wird. Von Lehrkräften wird erwartet, dass sie empirische Daten über ihren eigenen Unterricht rezipieren und Konsequenzen für die Unterrichtsentwicklung ableiten. Für die geringe Wirksamkeit dieses Ansatzes mehren sich allerdings inzwischen die theoretischen und empirischen Belege (Maier 2008). Es bleibt eine wichtige Frage, wie Instrumente der kompetenzorientierten Rückmeldung von Leistungsdaten in der Praxis genutzt werden können. Hierzu analysiert der Beitrag von Spoden, Fleischer und Leutner in diesem Heft die Frage, welchen Einfluss die Auswertungsobjektivität von Lehrkräften auf die Kompetenzmodellierung bei zentralen Lernstandserhebungen hat.

Auch künftig wird die am Kompetenzkonzept orientierte Forschung Erkenntnisse zu theoretischen Modellierungen, zu methodischen Ansätzen und zur Verbesserung der Praxis des Mathematikunterrichts hervorbringen. Die dabei entwickelten Ansätze und Standards für die Erfassung mathematischer Kompetenzen sind bereits jetzt nicht mehr aus der Forschung wegzudenken. Dennoch bleibt die Kompetenzmodellierung weiterhin nur eines unter vielen Instrumenten zur empirischen Untersuchung mathematischer Leistungen. Insbesondere zur Aufklärung von Denk- oder Lernprozessen sind Instrumente der Erfassung interindividueller Unterschiede nicht ausreichend, sondern müssen um experimentelle und hermeneutische Ansätze ergänzt werden.

In welcher Weise und in welchem Umfang konkrete Kompetenzmodellierungen Einzug ins Klassenzimmer halten werden, ist zurzeit noch offen. Auf Seiten der For- 
schung hängt dies davon ab, inwiefern die entwickelten bzw. noch zu entwickelnden Kompetenzmodelle bestehende diagnostische Verfahren für mathematische Leistungen (Hasselhorn et al. 2013) ergänzen können. Klassische Tests und qualitative Ansätze der mathematischen Diagnostik (z.B. Wollring et al. 2011) sind einstweilen nicht zu ersetzen. Einige Projekte außerhalb des deutschen Raumes zeigen aber auf, wie moderne Psychometrie und mathematikdidaktische Expertise zusammenwirken können, um praxistaugliche Instrumente zu generieren (z.B. Stacey und Steinle 2006).

Das Journal für Mathematikdidaktik wird weiterhin eine Plattform für die Darstellung von fachbezogenen Arbeiten im Bereich der Kompetenzforschung und für den wissenschaftlichen Diskurs zu diesem Bereich bieten.

\section{Rolf Biehler, Timo Leuders als Themenschwerpunktherausgeber}

\section{Literatur}

Altrichter, H., \& Heinrich, M. (2006). Evaluation als Steuerungsinstrument im Rahmen eines „,neuen Steuerungsmodells“ im Schulwesen. In W. Böttcher (Hrsg.), Evaluation im Bildungswesen: Eine Einführung in Grundlagen und Praxisbeispiele (S. 51-64). Weinheim: Juventa-Verlag.

Baumert, J., Klieme, E., Neubrand, M., Prenzel, M., Schiefele, U., Schneider, W., Stanat, P., Tillmann, J., \& Weiss, M. (Hrsg.) (2001). PISA 2000: Basiskompetenzen von Schülerinnen und Schülern im internationalen Vergleich. Opladen: Leske \& Budrich.

Böttcher, W. (2002). Für ein verbindliches Kerncurriculum an Grundschulen. In W. Böttcher \& P. E. Kalb (Hrsg.), Kerncurriculum: Was Kinder in der Grundschule lernen sollen (S. 14-37). Weinheim: Beltz.

Hartig, J. (2008). Psychometric models for the assessment of competencies. In J. Hartig, E. Klieme, \& D. Leutner (Hrsg.), Assessment of competencies in educational contexts (S. 69-90). Cambridge: Hogrefe.

Hartig, J., Klieme, E., \& Leutner, D. (Hrsg.) (2008). Assessment of competencies in educational contexts. Cambridge: Hogrefe.

Hasselhorn, M., Heinze, A., Schneider, W., \& Trautwein, U. (Hrsg.) (2013). Diagnostik mathematischer Kompetenzen. Göttingen: Hogrefe Verlag.

Helmke, A., Hosenfeld, I., \& Schrader, F.-W. (2004). Vergleichsarbeiten als Instrument zur Verbesserung der Diagnosekompetenz von Lehrkräften. In R. Arnold \& C. Griese (Hrsg.), Schulleitung und Schulentwicklung (S. 119-143). Hohengehren: Schneider.

Heymann, H.-W. (1996). Allgemeinbildung und Mathematik. Weinheim: Beltz.

Klieme, E., \& Leutner, D. (2006). Kompetenzmodelle zur Erfassung individueller Lernergebnisse und zur Bilanzierung von Bildungsprozessen: Beschreibung eines neu eingerichteten Schwerpunktprogramms der DFG. Zeitschrift für Pädagogik, 52, 876-903.

Klieme, E., Avenarius, H., Blum, W., et al. (2003). Zur Entwicklung nationaler Bildungsstandards: Eine Expertise. Bonn: BMBF.

Leuders, T. (2011). Kompetenzorientierung - Eine Chance für die Weiterentwicklung des Mathematikunterrichts? In K. Eilerts, A. Hilligus, G. Kaiser, \& P. Bender (Hrsg.), Kompetenzorientierung in Schule und Lehrerbildung: Perspektiven der bildungspolitischen Diskussion, der Bildungsforschung und der Lehrerbildung. Festschrift für Hans-Dieter Rinkens (S. 285-303). Münster: Lit-Verlag.

Leuders, T., Barzel, B., \& Hußmann, S. (2005). Outcome standards and core curricula: a new orientation for mathematics teachers in Germany. ZDM. Zentralblatt für Didaktik der Mathematik, 37(4), 275286.

Maier, U. (2008). Rezeption und Nutzung von Vergleichsarbeiten aus der Perspektive von Lehrkräften. Zeitschrift für Pädagogik, 54(1), 95-117.

Niss, M. (2003). Mathematical competencies and the learning of mathematics: the Danish KOM project. In A. Gagatsis \& S. Papastavridis (Hrsg.), 3rd Mediterranean conference on mathematical education (S. 115-123). Athen: Hellenic Mathematical Society.

OECD (1999). Measuring student knowledge and skills—a new framework for assessment. Paris: OECD. 
Pellegrino, J., Chudowsky, N., \& Glaser, R. (Hrsg.) (2001). Knowing what students know: the science and design of educational assessment. Washington: Academy Press.

Rost, J. (2004). Lehrbuch Testtheorie - Testkonstruktion (2. Aufl.). Bern: Huber.

Rupp, A., Templin, J., \& Henson, R. (2010). In Diagnostic measurement: theory, methods, and applications. New York: Guilford.

Stacey, K., \& Steinle, V. (2006). A case of the inapplicability of the rasch model: mapping conceptual learning. Mathematics Education Research Journal, 18(2), 77-92.

Weinert, F. E. (2001). Concept of competence: a conceptual clarification. In D. Rychen \& L. Salganik (Hrsg.), Defining and selecting key competencies (S. 45-66). Seattle: Hogrefe \& Huber.

Wollring, B., Peter-Koop, A., Haberzettl, N., Becker, N., \& Spindeler, B. (2011). Elementarmathematisches Basisinterview Größen und Messen, Raum und Form. Offenburg: Mildenberger. 